\title{
Instanton contribution to the Sivers function
}

\author{
I.O. Cherednikov ${ }^{\mathrm{a}, \mathrm{b}, \mathrm{c}, 1}$, U. D’Alesio $^{\mathrm{b}}$, N.I. Kochelev ${ }^{\mathrm{c}, \mathrm{d}}$, F. Murgia ${ }^{\mathrm{b}, *}$ \\ ${ }^{a}$ Institut für Theoretische Physik II, Ruhr-Universität Bochum, D-44780 Bochum, Germany \\ ${ }^{\mathrm{b}}$ Dipartimento di Fisica, Università di Cagliari and INFN, Sezione di Cagliari, C.P. 170, I-09042 Monserrato (CA), Italy \\ c Joint Institute for Nuclear Research, Bogoliubov Laboratory of Theoretical Physics, Moscow Region, 141980 Dubna, Russia \\ d School of Physics and Astronomy and Center for Theoretical Physics, Seoul National University, Seoul 151-747, South Korea
}

Received 6 July 2006; accepted 1 September 2006

Available online 27 September 2006

Editor: G.F. Giudice

\begin{abstract}
We study the Sivers function for valence $u$ and $d$ quarks in the proton within the instanton model for QCD vacuum, adopting the MIT bag model wave functions for quarks. Within approaches based on perturbative one-gluon final state interactions a non-zero value of the Sivers function is related to the presence of both $S$ and $P$ wave components in quark wave functions. We show that the instanton-induced chromomagnetic, nonperturbative interaction leads to very specific spin-spin correlations between the struck and spectator quarks, resulting in a non-trivial flavour dependence of the Sivers function. Comparison of the obtained Sivers functions with phenomenological parameterizations is discussed. (c) 2006 Elsevier B.V. All rights reserved.
\end{abstract}

\section{Introduction}

Understanding the spin structure of the nucleon is a long-standing, challenging problem for strong interaction theories which has received renewed interest in recent years. The investigation of mechanisms able to explain the unambiguous observation of large transverse single spin asymmetries (SSA) in inclusive meson production in hadronic collisions and in semi-inclusive deep inelastic scattering (SIDIS) may help in understanding the origin of spin effects in hadronic physics. Recently, significant SSA's in pion electroproduction on both longitudinally [1], and transversely [2] polarized proton targets have been observed by the HERMES Collaboration at DESY. Analogous measurements and processes are actively under investigation by the COMPASS experiment at CERN [3]. Measurements of the SSA for pion production in polarized proton-proton collisions, both in the central rapidity region and at large Feynman $x_{F}$, moderately large transverse momentum and center of mass energy $\sqrt{s}=200 \mathrm{GeV}$, have been performed by the PHENIX [4], STAR [5] and BRAHMS [6] Collaborations at the Relativistic Heavy Ion Collider (RHIC) at Brookhaven. These experiments plan to refine their measurements, improving the statistics and enlarging the kinematical region explored.

We should emphasize that in spite of the fact that large, "anomalous", SSA at high energy and large transverse momentum were discovered long time ago in both inclusive and exclusive hadron production in hadron-hadron collisions [7], their understanding within QCD [8-10], although significantly improved in recent years [11,12], is far from being complete, see e.g. Ref. [13].

In perturbative QCD (pQCD) approaches with inclusion of intrinsic (or primordial) parton motion, SSA can be related to the so-called naively T-odd, transverse momentum dependent (TMD), Sivers distribution and Collins fragmentation functions [14-16]. These functions provide very important information on the behaviour of strong interactions at large distances and can be calculated only within nonperturbative QCD. Therefore, in the absence of first-principles QCD calculations for these functions, e.g., within

\footnotetext{
* Corresponding author.

E-mail addresses: igor.cherednikov@jinr.ru (I.O. Cherednikov), umberto.dalesio@ca.infn.it (U. D’Alesio), kochelev@ theor.jinr.ru (N.I. Kochelev), francesco.murgia@ca.infn.it (F. Murgia).

1 Alexander von Humboldt fellow.
} 
lattice QCD, it is very important to calculate SSA within some suitable model accounting for nonperturbative QCD effects. In the last years it has been shown that the instanton model (IM) can well describe the main nonperturbative aspects of QCD [17-21]. The first attempts to apply the IM to the study of SSA were done in Refs. [22-25]. In particular, the instanton induced quark-quark interaction was shown to be important for the appearance of significant SSA in quark-quark scattering [22,23]. In Ref. [25] this mechanism has been applied to estimate the current jet single spin asymmetry in SIDIS, within a modified version of the final-state interaction approach suggested in Ref. [11]. The contribution to the SSA in SIDIS coming from the instanton-induced spin-flip photon-quark vertex is the subject of a recent paper [24].

In this Letter we derive for the first time the Sivers distributions for $u$ and $d$ valence quarks inside a proton, within the instanton model for QCD vacuum and using the MIT bag model for quark wave functions. Part of the calculation is performed along the lines of Ref. [26], where however the perturbative one-gluon exchange contribution to the Sivers function was considered. Analogous calculations, using a quark-diquark model for the nucleon, were presented in Refs. [27,28].

\section{Sivers function within the MIT bag model formalism}

The Sivers function [8], $f_{1 T}^{\perp \alpha}\left(x, \boldsymbol{k}_{\perp}^{2}\right)$ or $\Delta^{N} f_{\alpha / p^{\uparrow}}\left(x, \boldsymbol{k}_{\perp}^{2}\right)$ (see Ref. [29] for details on different notations adopted in the literature), describes the correlation between the intrinsic transverse momentum of an unpolarized quark (with flavour $\alpha$ ) and the parent proton transverse polarization. The general correlator for transverse momentum dependent quark distribution functions is given by [14]

$$
\Phi^{\alpha}\left(x, \boldsymbol{k}_{\perp}, S\right)=\left.\int \frac{d \xi^{-} d^{2} \xi_{\perp}}{(2 \pi)^{3}} \mathrm{e}^{-i k \cdot \xi}\left\langle P, S\left|\bar{\psi}^{\alpha}(\xi) V^{\dagger}(\xi) V(0) \psi^{\alpha}(0)\right| P, S\right\rangle\right|_{\xi^{+}=0},
$$

where $k^{\mu}=x P^{\mu}+k_{\perp}^{\mu}$ is the quark four-momentum, and $P, S$ are the hadron momentum and spin vector respectively. The Wilson lines, also called gauge-links, $V(\xi)$, are path-ordered exponentials required to preserve gauge invariance; as it was shown recently, they are also crucial in order to get non-vanishing $T$-odd TMD parton distributions, like the Sivers function [11,12]. They are defined as follows:

$$
V(\xi)=\mathcal{P} \exp \left(-i g_{s} \int_{\xi^{-}}^{\infty} d z^{-} \hat{A}^{+}\left(z^{-}, \xi_{\perp}\right)\right) \cdot \mathcal{P} \exp \left(-i g_{s} \int_{\xi_{\perp}}^{\infty} d z_{\perp} \hat{A}_{\perp}\left(z^{-}=\infty, z_{\perp}\right)\right),
$$

where $g_{s}$ is the strong coupling constant, $\hat{A}^{\mu}=\sum_{a} t^{a} A_{a}^{\mu}, t^{a}=\lambda^{a} / 2$ are the Gell-Mann colour matrices and $A_{a}^{\mu}$ are the gluon gauge fields. In the following, we will work in a covariant gauge, so that we can neglect the second term in Eq. (2), which vanishes in non-singular gauges [30].

TMD distribution functions can be obtained by first opportunely Dirac-projecting and then taking the trace of the general correlator, Eq. (1). In the case of interest, for example, the Sivers function for a quark with flavour $\alpha$ can be extracted as follows:

$$
f_{1 T}^{\perp \alpha}\left(x, k_{\perp}\right)=\frac{1}{2} \frac{M_{P}}{2 k_{y}} \operatorname{Tr}\left[\gamma^{+}\left[\Phi^{\alpha}\left(x, \boldsymbol{k}_{\perp}, S_{\perp x}\right)-\Phi^{\alpha}\left(x, \boldsymbol{k}_{\perp},-S_{\perp x}\right)\right]\right],
$$

where $k_{\perp}=\left|\boldsymbol{k}_{\perp}\right|, M_{P}$ is the proton mass and, to be definite, we have considered a proton moving along the $+z$-direction and transversely polarized along the $x$-direction; in this way, we have also subtracted the $\boldsymbol{k}_{\perp}$-even contribution. Due to translational invariance, using Eq. (1) one can rewrite the last equation in the form

$$
f_{1 T}^{\perp \alpha}\left(x, k_{\perp}\right)=\frac{M_{P} E_{P}}{k_{y}} \int \frac{d \xi^{-} d^{2} \xi_{\perp}}{(2 \pi)^{3}} \mathrm{e}^{-i k \cdot \xi} \int d^{3} \boldsymbol{r}\left\langle\uparrow_{x}\left|\bar{\psi}^{\alpha}(\xi+\boldsymbol{r}) V^{\dagger}(\xi+\boldsymbol{r}) \gamma^{+} V(\boldsymbol{r}) \psi^{\alpha}(\boldsymbol{r})\right| \uparrow_{x}\right\rangle .
$$

Hereafter, for shortness, we will indicate by $\left\langle\uparrow_{x}|\cdots| \uparrow_{x}\right\rangle$ any matrix element of the form $\left[\left\langle S_{\perp x}|\cdots| S_{\perp x}\right\rangle-\left\langle-S_{\perp x}\right| \cdots\right.$ $\left.\left|-S_{\perp x}\right\rangle\right] / 2$.

In order to estimate the instanton contribution to the Sivers function, following Ref. [26] we will use (valence) quark wave functions from the MIT bag model. The Fourier transform of the lowest modes of these functions, to which we limit, is then defined as

$$
\psi^{\alpha, i}(\xi)=\sum_{m} \int \frac{d^{3} \boldsymbol{k}}{(2 \pi)^{3}} \phi_{m}(\boldsymbol{k}) \mathrm{e}^{-i k \cdot \xi} a_{\alpha, m}^{i},
$$

where $a_{\alpha, m}^{i}$ is the quark annihilation operator, $\alpha, i$, are respectively the quark flavour and colour indexes and $m$ is its spin component (helicity), $m= \pm 1 / 2$. Since we consider only valence quarks in the proton, a similar term related to antiquark operators is omitted. Moreover, in the MIT bag model

$$
\phi_{m}(\boldsymbol{k})=N \sqrt{4 \pi} R_{0}^{3}\left(\begin{array}{c}
t_{0}(k) \chi_{m} \\
\boldsymbol{\sigma} \cdot \hat{\boldsymbol{k}} t_{1}(k) \chi_{m}
\end{array}\right),
$$




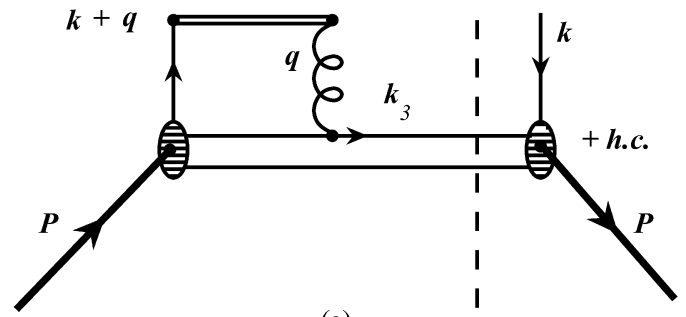

(a)

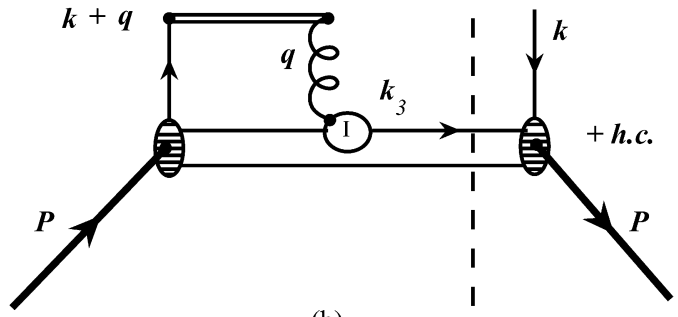

(b)

Fig. 1. Perturbative (a) and instanton induced (b) contributions to the Sivers function. The symbol $I$ denotes the instanton.

where $k=|\boldsymbol{k}|, \hat{\boldsymbol{k}}=\boldsymbol{k} / k$,

$$
t_{i}(k)=\int_{0}^{1} d x x^{2} j_{i}\left(x k R_{0}\right) j_{i}(x \omega), \quad N^{2}=\frac{1}{R_{0}^{3}} \frac{\omega^{3}}{2(\omega-1) \sin ^{2} \omega},
$$

$R_{0}$ is the bag (confinement) radius, and $\omega=2.043$ for the lowest state of massless quarks inside the bag. In the proton rest frame $E_{P}=M_{P}$ and the bag radius $R_{0}=4 \omega / E_{P}$ is fixed by the bag stability condition, $d E_{P} / d R=0$.

The transversely polarized proton (e.g., in the $\pm x$-direction) is described by the state vector

$$
\left|S_{ \pm x}\right\rangle=\frac{1}{\sqrt{2}}\left(\left|S_{+z}\right\rangle \pm\left|S_{-z}\right\rangle\right)
$$

where the longitudinally polarized states read

$$
\begin{aligned}
& \left|S_{+z}\right\rangle=\frac{1}{\sqrt{6}} \frac{1}{\sqrt{18}} \epsilon_{i j k} \epsilon_{n_{1}, n_{2}, n_{3}}\left[a_{u^{\uparrow}}^{i \dagger}\left(n_{1}\right) a_{u^{\dagger}}^{j \dagger}\left(n_{2}\right) a_{d^{\downarrow}}^{k \dagger}\left(n_{3}\right)-a_{u^{\uparrow}}^{i \dagger}\left(n_{1}\right) a_{u^{\downarrow}}^{j \dagger}\left(n_{2}\right) a_{d^{\dagger}}^{k \dagger}\left(n_{3}\right)\right]|0\rangle, \\
& \left|S_{-z}\right\rangle=\frac{1}{\sqrt{6}} \frac{1}{\sqrt{18}} \epsilon_{i j k} \epsilon_{n_{1}, n_{2}, n_{3}}\left[-a_{u^{\downarrow}}^{i \dagger}\left(n_{1}\right) a_{u^{\prime}}^{j \dagger}\left(n_{2}\right) a_{d^{\dagger}}^{k \dagger}\left(n_{3}\right)+a_{u^{\dagger}}^{i \dagger}\left(n_{1}\right) a_{u \downarrow}^{j \dagger}\left(n_{2}\right) a_{d^{\dagger}}^{k \dagger}\left(n_{3}\right)\right]|0\rangle,
\end{aligned}
$$

and the $n_{i}$ are position indexes.

\section{Instanton contribution}

The contribution to the quark Sivers function coming from perturbative, one-gluon exchange, final state interactions (see Fig. 1a) has been considered in Ref. [26] in the framework of the MIT bag model. The interaction Lagrangian in this case is simply given by

$$
\mathcal{L}_{\text {int }}^{P}=-\sum_{\beta} \sum_{a, k, l} g_{s} \bar{\psi}^{\beta, k}(\eta) \gamma^{\mu} t_{k l}^{a} \psi^{\beta, l}(\eta) A_{a \mu}(\eta),
$$

where $\beta$ and $a, k, l$ are respectively flavour and colour indexes. It has been shown in Ref. [26] that the interference between $S$ and $P$ wave components of the bag quark wave functions leads to a non-zero value of the Sivers function. Clearly, these contributions are related to the dynamics of quark confinement at the scale of a typical hadronic size; as such, they can be responsible for the SSA at relatively small transverse momentum, $k_{\perp} \approx 1 / R_{0} \approx 200-300 \mathrm{MeV} / c$.

In this Letter we consider the contribution to the Sivers function which stems from a completely different QCD dynamics (see Fig. 1b), namely from the nonperturbative chromomagnetic quark-gluon interaction induced by instantons [22]

$$
\mathcal{L}_{\text {int }}^{N P}=i \frac{\pi^{2} f}{g_{s}} \sum_{\beta} \sum_{a, k, l} \frac{1}{m_{\beta}^{*}} \bar{\psi}^{\beta, k}(\eta) \sigma_{\mu \nu} t_{k l}^{a} \psi^{\beta, l}(\eta) G_{a}^{\mu \nu}(\eta),
$$

where $f=\pi^{2} n_{c} \rho_{c}^{4}$ is the so-called packing fraction of instantons in the QCD vacuum, $n_{c}$ is the instanton density, $\rho_{c}$ is the average instanton size, $m_{u}^{*} \approx m_{d}^{*} \approx m^{*}$ is some appropriate mass parameter for the quark propagator in the instanton vacuum [17] and $\sigma_{\mu \nu}=\left[\gamma_{\mu}, \gamma_{\nu}\right] / 2$. In mean field approximation for free quarks $m^{*}$ has the meaning of an effective quark mass (see, e.g., the recent discussion in Ref. [31]). For off-shell quarks with virtualities $k_{1}$ and $k_{2}$ and a gluon with virtuality $q$ the instanton induced quarkgluon vertex should be multiplied by the factor

$$
F\left(k_{1}, k_{2}, q\right)=\Phi\left(k_{1} \rho_{c} / 2\right) \Phi\left(k_{2} \rho_{c} / 2\right) G\left(q \rho_{c}\right),
$$

where

$$
\Phi(z)=-z \frac{d}{d z}\left[I_{0}(z) K_{0}(z)-I_{1}(z) K_{1}(z)\right], \quad G(z)=\frac{4}{z^{2}}-2 K_{2}(z)
$$


are the Fourier-transformed quark zero-mode and instanton fields respectively and $I_{v}(z), K_{v}(z)$, are the well-known modified Bessel functions. The main feature of the instanton vertex, Eq. (11), in comparison with the perturbative one, Eq. (10), is the presence of a quark spin-flip contribution. Moreover, the strong coupling constant $g_{s}$ enters the denominator of the effective strength in Eq. (11); therefore there is not any $\alpha_{s}$ power suppression of the spin-flip contributions in our nonperturbative approach to SSA. We should also mention that the average instanton size is much smaller than the confinement radius, $\rho_{c} \approx(1 / 3) R_{\text {conf }}$, so that the instanton contribution to the SSA should be significant at larger momentum transfer in comparison with the contribution coming from quark bound-state dynamics.

Let us now estimate the contribution of the chromomagnetic interaction to the quark Sivers function coming from Fig. $1 \mathrm{~b}$. Inserting Eq. (11) and the gauge links, Eq. (2), into Eq. (4), and taking the leading term we find

$$
\begin{aligned}
f_{1 T}^{\perp \alpha}\left(x, k_{\perp}\right)_{I}= & -i \frac{M_{P} E_{P}}{k_{y}} \frac{\pi^{2} f}{m^{*}} \int \frac{d \xi^{-} d^{2} \xi_{\perp}}{(2 \pi)^{3}} \mathrm{e}^{-i k \cdot \xi} \int d^{3} \boldsymbol{r} \int_{\xi^{-}}^{\infty} d z^{-} \int d^{4} \eta \\
& \times\left\langle\uparrow_{x}\right| \bar{\psi}^{\alpha, i}(\xi+\boldsymbol{r}) A_{a}^{+}\left(z^{-}, \xi_{\perp}+\boldsymbol{r}\right) t_{i j}^{a} \gamma^{+} \psi^{\alpha, j}(\boldsymbol{r}) \\
& \times \sum_{\beta} \bar{\psi}^{\beta, k}(\eta) \sigma_{\mu \nu} t_{k l}^{b} \psi^{\beta, l}(\eta)\left[\partial^{\mu} A_{b}^{v}(\eta)-\partial^{\nu} A_{b}^{\mu}(\eta)\right]\left|\uparrow{ }_{x}\right\rangle+\text { h.c. }
\end{aligned}
$$

As one can see, the dependence on the strong coupling constant disappears and the instanton contribution to the Sivers function is proportional to the packing fraction of instantons in the vacuum. We now use Eq. (5) for the quark fields; by taking also the Fourier transforms of the gluon fields, using the perturbative gluonic propagator (given consistently in a covariant gauge, see comments after Eq. (2)) as an approximation for the correlator of two gauge fields,

$$
\left\langle\tilde{A}_{a}^{+}(q) \tilde{A}_{b}^{v}(p)\right\rangle=(2 \pi)^{4} \delta^{(4)}(q+p)\left(\frac{-i g^{+v} \delta_{a b}}{q^{2}}\right),
$$

and using Eq. (12) where appropriate, we get

$$
\begin{aligned}
f_{1 T}^{\perp \alpha}\left(x, k_{\perp}\right)_{I}= & -\frac{M_{P} E_{P}}{k_{y}} \frac{\pi^{2} f}{m^{*}} \int \frac{d^{4} q}{(2 \pi)^{4}} G\left(q \rho_{c}\right) \frac{1}{q^{2}} \frac{1}{q^{+}+i \epsilon} \int \frac{d^{3} \boldsymbol{k}_{1}}{(2 \pi)^{3}} \int \frac{d^{3} \boldsymbol{k}_{3}}{(2 \pi)^{3}} \\
& \times 2 \pi \delta\left(q^{0}\right) \delta\left(k^{+}-q^{+}-k_{1}^{+}\right) \delta^{(2)}\left(\boldsymbol{k}_{\perp}-\boldsymbol{q}_{\perp}-\boldsymbol{k}_{1 \perp}\right) \\
& \times \sum_{m_{i}} \sum_{\beta} \sum_{a i j k l} t_{i j}^{a} t_{k l}^{a}\left\langle\uparrow_{x}\left|a_{\alpha, m_{1}}^{i \dagger} a_{\alpha, m_{2}}^{j} a_{\beta, m_{3}}^{k \dagger} a_{\beta, m_{4}}^{l}\right| \uparrow_{x}\right\rangle \\
& \times \phi_{m_{1}}^{\dagger}\left(\boldsymbol{k}_{1}\right) \gamma^{0} \gamma^{+} \phi_{m_{2}}\left(\boldsymbol{k}_{1}+\boldsymbol{q}\right) \phi_{m_{3}}^{\dagger}\left(\boldsymbol{k}_{3}\right) \gamma^{0}\left(q \gamma^{+}-\gamma^{+} \phi\right) \phi_{m_{4}}\left(\boldsymbol{k}_{3}-\boldsymbol{q}\right)+\text { h.c. }
\end{aligned}
$$

Concerning the Hermitian conjugate contribution, one can easily see that it can be obtained by simply exchanging $q \leftrightarrow-q$ in the first term of the previous equation. Taking also into account the relation

$$
\frac{1}{q^{+}-i \epsilon}-\frac{1}{q^{+}+i \epsilon}=i(2 \pi) \delta\left(q^{+}\right),
$$

performing the integrations over coordinates, and making use of the Dirac delta functions, one can simplify the sum of the two contributions, which finally reads

$$
\begin{aligned}
f_{1 T}^{\perp \alpha}\left(x, k_{\perp}\right)_{I}= & i \frac{M_{P} E_{P}}{k_{y}} \frac{\pi^{2} f}{m^{*}} \sqrt{2} \int \frac{d^{2} \boldsymbol{q}_{\perp} G\left(q_{\perp} \rho_{c}\right)}{(2 \pi)^{2}} \int \frac{d^{3} \boldsymbol{k}_{1}}{(2 \pi)^{3}} \int \frac{d^{3} \boldsymbol{k}_{3}}{(2 \pi)^{3}} \frac{1}{\boldsymbol{q}_{\perp}^{2}} \\
& \times \delta\left(k^{+}-k_{1}^{+}-q^{+}\right) \delta^{(2)}\left(\boldsymbol{k}_{\perp}-\boldsymbol{k}_{1 \perp}-\boldsymbol{q}_{\perp}\right) \\
& \times \sum_{m_{i}} T_{\{m\}}^{\alpha} \phi_{m_{1}}^{\dagger}\left(\boldsymbol{k}_{1}\right) \gamma^{0} \gamma^{+} \phi_{m_{2}}\left(\boldsymbol{k}_{1}+\boldsymbol{q}_{\perp}\right) \phi_{m_{3}}^{\dagger}\left(\boldsymbol{k}_{3}\right) \Gamma\left(\boldsymbol{q}_{\perp}\right) \phi_{m_{4}}\left(\boldsymbol{k}_{3}-\boldsymbol{q}_{\perp}\right),
\end{aligned}
$$

where $q_{\perp}=\left|\boldsymbol{q}_{\perp}\right|$,

$$
\begin{aligned}
& T_{\{m\}}^{\alpha}=\sum_{\beta} \sum_{a i j k l} t_{i j}^{a} t_{k l}^{a}\left\langle\uparrow_{x}\left|a_{\alpha, m_{1}}^{i \dagger} a_{\alpha, m_{2}}^{j} a_{\beta, m_{3}}^{k \dagger} a_{\beta, m_{4}}^{l}\right| \uparrow_{x}\right\rangle \\
& \quad=\frac{1}{2} \sum_{\beta} \sum_{a i j k l} t_{i j}^{a} t_{k l}^{a}\left(\left\langle S_{+z}\left|a_{\alpha, m_{1}}^{i \dagger} a_{\alpha, m_{2}}^{j} a_{\beta, m_{3}}^{k \dagger} a_{\beta, m_{4}}^{l}\right| S_{-z}\right\rangle+\left\langle S_{-z}\left|a_{\alpha, m_{1}}^{i \dagger} a_{\alpha, m_{2}}^{j} a_{\beta, m_{3}}^{k \dagger} a_{\beta, m_{4}}^{l}\right| S_{+z}\right\rangle\right), \\
& \Gamma\left(\boldsymbol{q}_{\perp}\right)=\gamma^{0}\left(\boldsymbol{\gamma} \cdot \boldsymbol{q}_{\perp} \gamma^{+}-\gamma^{+} \boldsymbol{\gamma} \cdot \boldsymbol{q}_{\perp}\right),
\end{aligned}
$$

and where $\alpha$ (not summed), $\beta=u, d$ are flavour indexes and $\{m\}$ stands for the set of quark helicity indexes $\left(m_{1}, m_{2}, m_{3}, m_{4}\right)$. 
It is useful to compare this result with the perturbative contribution, Ref. [26]:

$$
f_{1 T}^{\perp \alpha}\left(x, k_{\perp}\right)_{\mathrm{pQCD}}=i 2 g_{s}^{2} \frac{M_{P} E_{P}}{k_{y}} \sum_{m_{i}} T_{\{m\}}^{\alpha} \int \frac{d^{2} \boldsymbol{q}_{\perp}}{(2 \pi)^{5}} \frac{1}{\boldsymbol{q}_{\perp}^{2}} F_{P m_{3}, m_{4}}^{s}\left(\boldsymbol{q}_{\perp}^{2}\right) F_{m_{1}, m_{2}}^{c}\left(\boldsymbol{q}_{\perp}, \boldsymbol{k}\right),
$$

where the current quark-gluon vertex contribution (common to both the perturbative and the instanton approach) is given by

$$
F_{m_{1}, m_{2}}^{c}\left(\boldsymbol{q}_{\perp}, \boldsymbol{k}\right)=\phi_{m_{1}}^{\dagger}\left(\boldsymbol{k}-\boldsymbol{q}_{\perp}\right) \gamma^{0} \gamma^{+} \phi_{m_{2}}(\boldsymbol{k})
$$

and consists of two terms, with and without helicity flip of the struck quark:

$$
\begin{aligned}
F_{m_{1}, m_{2}}^{c}\left(\boldsymbol{q}_{\perp}, \boldsymbol{k}\right)= & \frac{1}{\sqrt{2}}\left(F_{m_{1}}^{c ; \text { even }} \delta_{m_{1}, m_{2}}+F_{m_{1}}^{c ; \text { odd }} \delta_{m_{1},-m_{2}}\right) \\
= & \frac{1}{\sqrt{2}} \tilde{N}^{2}\left\{\left[t_{0}(k) t_{0}\left(k^{\prime}\right)+\frac{k_{z}}{k} t_{1}(k) t_{0}\left(k^{\prime}\right)\right.\right. \\
& \left.+\frac{k_{z}}{k^{\prime}} t_{0}(k) t_{1}\left(k^{\prime}\right)+\frac{1}{k k^{\prime}}\left[\boldsymbol{k} \cdot \boldsymbol{k}^{\prime}+i 2 m_{1}\left(k_{x} q_{\perp y}-k_{y} q_{\perp x}\right)\right] t_{1}(k) t_{1}\left(k^{\prime}\right)\right] \delta_{m_{1}, m_{2}} \\
& +\left[\frac{\left(2 m_{1} k_{x}-i k_{y}\right)}{k} t_{1}(k) t_{0}\left(k^{\prime}\right)-\frac{\left(2 m_{1} k_{x}^{\prime}-i k_{y}^{\prime}\right)}{k^{\prime}} t_{0}(k) t_{1}\left(k^{\prime}\right)\right. \\
& \left.\left.+\frac{k_{z}\left(2 m_{1} q_{\perp x}-i q_{\perp y}\right)}{k k^{\prime}} t_{1}(k) t_{1}\left(k^{\prime}\right)\right] \delta_{m_{1},-m_{2}}\right\},
\end{aligned}
$$

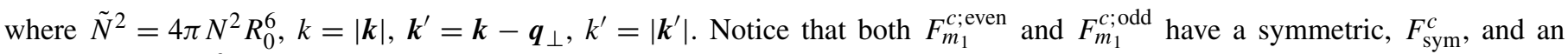
antisymmetric, $F_{\text {antisym }}^{c}$, component for $m_{1} \leftrightarrow-m_{1}$.

The (perturbative) spectator quark-gluon vertex contribution reads

$$
F_{P m_{3}, m_{4}}^{s}\left(\boldsymbol{q}_{\perp}^{2}\right)=\int \frac{d^{3} \boldsymbol{k}_{3}}{(2 \pi)^{3}} \phi_{m_{3}}^{\dagger}\left(\boldsymbol{k}_{3}\right) \gamma^{0} \gamma^{+} \phi_{m_{4}}\left(\boldsymbol{k}_{3}-\boldsymbol{q}_{\perp}\right)=\frac{1}{\sqrt{2}} F_{P}^{s}\left(\boldsymbol{q}_{\perp}^{2}\right) \delta_{m_{3}, m_{4}},
$$

where the spectator function $F_{P}^{s}\left(\boldsymbol{q}_{\perp}^{2}\right)$ is given by (see also Ref. [26])

$$
\begin{aligned}
F_{P}^{S}\left(\boldsymbol{q}_{\perp}^{2}\right)= & \frac{\tilde{N}^{2}}{(2 \pi)^{2}} \int \kappa^{2} d \kappa d(\cos \theta)\left\{t_{0}(\kappa) t_{0}\left(\kappa^{\prime}\right)+\frac{\kappa \cos \theta-q_{\perp}}{\kappa^{\prime}} t_{0}(\kappa) t_{1}\left(\kappa^{\prime}\right)\right. \\
& \left.+\cos \theta t_{1}(\kappa) t_{0}\left(\kappa^{\prime}\right)+\frac{\kappa-q_{\perp} \cos \theta}{\kappa^{\prime}} t_{1}(\kappa) t_{1}\left(\kappa^{\prime}\right)\right\}
\end{aligned}
$$

and we have defined $\boldsymbol{\kappa}=\boldsymbol{k}_{3}, \kappa=\left|\boldsymbol{k}_{3}\right|, \boldsymbol{\kappa}^{\prime}=\boldsymbol{k}_{3}-\boldsymbol{q}_{\perp}, \kappa^{\prime}=\left|\boldsymbol{\kappa}^{\prime}\right|=\left[\kappa^{2}+q_{\perp}^{2}-2 \kappa q_{\perp} \cos \theta\right]^{1 / 2}$.

It is worth to emphasize at this point that, according to our definition of the matrix elements $\left\langle\uparrow_{x}|\cdots| \uparrow_{x}\right\rangle$ (see comments after Eqs. (4) and (19)) the only non-vanishing $T_{m_{1}, m_{2}, m_{3}, m_{4}}^{\alpha}$ are those of the form $T_{m_{1}, m_{1}, m_{3},-m_{3}}^{\alpha}$ or $T_{m_{1},-m_{1}, m_{3}, m_{3}}^{\alpha}$. Therefore, one-gluon exchange contributions, associated either with the perturbative or the nonperturbative spectator vertex, can be non-vanishing only when we have just (and at least) one helicity-flip term, either in the struck-quark vertex, or in the spectator one, but not in both of them. In the perturbative case, the struck-quark helicity-flip situation takes place, Ref. [26]. As we will see below, the specific instanton-induced spin-spin correlation allows both the struck- and spectator-quark helicity-flip mechanisms to contribute.

In the nonperturbative case, it is convenient to define an instanton analogue of the function $F_{P}^{S}\left(\boldsymbol{q}_{\perp}^{2}\right)$ :

$$
F_{I m_{3}, m_{4}}^{s}\left(\boldsymbol{q}_{\perp}\right)=\int \frac{d^{3} \boldsymbol{k}_{3}}{(2 \pi)^{3}} \phi_{m_{3}}^{\dagger}\left(\boldsymbol{k}_{3}\right) \Gamma\left(\boldsymbol{q}_{\perp}\right) \phi_{m_{4}}\left(\boldsymbol{k}_{3}-\boldsymbol{q}_{\perp}\right)=\frac{1}{\sqrt{2}}\left(F_{I m_{3}}^{s ; \text { even }}\left(\boldsymbol{q}_{\perp}\right) \delta_{m_{3}, m_{4}}+F_{I m_{3}}^{s ; \text { odd }}\left(\boldsymbol{q}_{\perp}\right) \delta_{m_{3},-m_{4}}\right)
$$

so that the instanton contribution to the Sivers function reads

$$
f_{1 T}^{\perp \alpha}\left(x, k_{\perp}\right)_{I}=i \frac{M_{P} E_{P}}{k_{y}} \frac{\pi^{2} f}{m^{*}} 2 \int \frac{d^{2} \boldsymbol{q}_{\perp}}{(2 \pi)^{5}} \frac{1}{\boldsymbol{q}_{\perp}^{2}} G\left(q_{\perp} \rho_{c}\right) \sum_{m_{i}} T_{\{m\}}^{\alpha} F_{m_{1}, m_{2}}^{c}\left(\boldsymbol{q}_{\perp}, \boldsymbol{k}\right) F_{I m_{3}, m_{4}}^{s}\left(\boldsymbol{q}_{\perp}\right) .
$$

Working in the standard representation of the Dirac matrices

$$
\boldsymbol{\gamma}=\left(\begin{array}{cc}
0 & \sigma \\
-\sigma & 0
\end{array}\right), \quad \gamma^{0} \gamma \gamma^{+}=\frac{1}{\sqrt{2}}\left(\begin{array}{cc}
-\sigma \sigma_{3} & -\sigma \\
\sigma & \sigma \sigma_{3}
\end{array}\right), \quad \gamma^{0} \gamma^{+} \gamma=\frac{1}{\sqrt{2}}\left(\begin{array}{cc}
-\sigma_{3} \sigma & \sigma \\
-\sigma & \sigma_{3} \sigma
\end{array}\right),
$$


one finds

$$
\begin{aligned}
F_{I m_{3}, m_{4}}^{s}\left(\boldsymbol{q}_{\perp}\right)= & \sqrt{2} \tilde{N}^{2} \int \frac{d^{3} \boldsymbol{\kappa}}{(2 \pi)^{3}} \chi_{m_{3}}^{\dagger}\left\{i\left[\boldsymbol{q}_{\perp} \times \boldsymbol{\sigma}\right]_{z} t_{0}(\kappa) t_{0}\left(\kappa^{\prime}\right)-\left(\boldsymbol{\sigma} \cdot \boldsymbol{q}_{\perp}\right)\left(\boldsymbol{\sigma} \cdot \boldsymbol{\kappa}^{\prime}\right) \frac{1}{\kappa^{\prime}} t_{0}(\kappa) t_{1}\left(\kappa^{\prime}\right)\right. \\
& \left.+(\boldsymbol{\sigma} \cdot \boldsymbol{\kappa})\left(\boldsymbol{\sigma} \cdot \boldsymbol{q}_{\perp}\right) \frac{1}{\kappa} t_{1}(\kappa) t_{0}\left(\kappa^{\prime}\right)-i(\boldsymbol{\sigma} \cdot \boldsymbol{\kappa})\left[\boldsymbol{q}_{\perp} \times \boldsymbol{\sigma}\right]_{z}\left(\boldsymbol{\sigma} \cdot \boldsymbol{\kappa}^{\prime}\right) \frac{1}{\kappa \kappa^{\prime}} t_{1}(\kappa) t_{1}\left(\kappa^{\prime}\right)\right\} \chi_{m_{4}} .
\end{aligned}
$$

After some algebra and taking into account symmetry properties of the integrand, one gets

$$
\begin{aligned}
& F_{I m_{3}}^{s ; \text { even }}\left(\boldsymbol{q}_{\perp}\right)=\frac{2 \tilde{N}^{2}}{(2 \pi)^{2}} \int \kappa^{2} d \kappa d(\cos \theta) q_{\perp}\left(\cos \theta t_{0}\left(\kappa^{\prime}\right) t_{1}(\kappa)-\frac{\kappa \cos \theta-q_{\perp}}{\kappa^{\prime}} t_{0}(\kappa) t_{1}\left(\kappa^{\prime}\right)\right) \\
& F_{I m_{3}}^{s ; \text { odd }}\left(\boldsymbol{q}_{\perp}\right)=\frac{2 \tilde{N}^{2}}{(2 \pi)^{2}}\left(2 m_{3} q_{\perp x}-i q_{\perp y}\right) \int \kappa^{2} d \kappa d(\cos \theta)\left(t_{0}(\kappa) t_{0}\left(\kappa^{\prime}\right)+\frac{\kappa-q_{\perp} \cos \theta}{\kappa^{\prime}} t_{1}(\kappa) t_{1}\left(\kappa^{\prime}\right)\right) .
\end{aligned}
$$

Notice that $F_{I m_{3}}^{s \text {; even }}$ is in fact independent of $m_{3}$, while $F_{I m_{3}}^{s ; \text { odd }}$ has both a symmetric and an antisymmetric component, $F_{I \text { sym }}^{s \text { odd }}$ and $F_{I \text { antisym }}^{s ; \text { odd }}$ for $m_{3} \leftrightarrow-m_{3}$.

This time we can have two additive contributions to the Sivers function: $F_{s ; \text { idd }}^{c \text { even }}$ and $F_{s ; \text { odd }}^{c \text {; even }}$, corresponding respectively to helicity-flip of the struck quark and no helicity-flip for the spectator quark, and vice versa,

$$
f_{1 T}^{\perp \alpha}\left(x, k_{\perp}\right)_{I} \propto \sum_{m_{1}, m_{3}}\left(F_{m_{1}}^{c ; \text { even }} F_{m_{3}}^{s ; \text { odd }} T_{m_{1}, m_{1}, m_{3},-m_{3}}^{\alpha}+F_{m_{1}}^{c ; \text { odd }} F_{m_{3}}^{s ; \text { even }} T_{m_{1},-m_{1}, m_{3}, m_{3}}^{\alpha}\right) .
$$

Therefore both the spectator and the current quark spin-flip terms contribute to the instanton-induced Sivers function. This is in contrast to the purely perturbative result, Eqs. (23) and (24), where only the current quark spin-flip gives a non-vanishing contribution to the Sivers function. Moreover, as we will see in a moment, it turns out that the instanton contribution has a completely different flavour dependence, due to the structure of the matrix element in Eq. (27). This could open a new way of understanding the unexpected flavour dependence of the Sivers function, as extracted from recent HERMES data [2] (see below).

An explicit calculation of the non-vanishing proton matrix elements $T_{\{m\}}^{\alpha}$ in Eq. (19) shows that they have the same value for any allowed combination of $m_{1}, m_{3}$, so that, e.g.,

$$
\begin{aligned}
F_{s ; \text { odd }}^{c ; \text { even }} & =\sum_{m_{1}, m_{3}} F_{m_{1}}^{c \text {;even }} F_{I m_{3}}^{s ; \text { odd }} T_{m_{1}, m_{1}, m_{3},-m_{3}}^{\alpha}=T_{\uparrow \uparrow \uparrow \downarrow}^{\alpha} \sum_{m_{1}, m_{3}} F_{m_{1}}^{c ; \text { even }} F_{I m_{3}}^{s ; \text { odd }} \\
& =\sum_{m_{1}, m_{3}} T_{m_{1}, m_{1}, m_{3},-m_{3}}^{\alpha}\left(\frac{1}{2} \sum_{m_{1}} F_{m_{1}}^{c ; \text { even }}\right)\left(\frac{1}{2} \sum_{m_{3}} F_{m_{3}}^{s ; \text { odd }}\right)=\left.C_{I}^{\alpha}\right|_{\text {odd }} ^{\text {even }} F_{\text {sym }}^{c ; \text { even }} F_{I \text { sym }}^{s ; \text { odd }},
\end{aligned}
$$

where $F_{\text {sym }}^{c \text {;even }}$ and $F_{I \text { sym }}^{s ; \text { odd }}$ can be obtained from Eqs. (23) and (31) respectively. Analogously,

$$
F_{s ; \text { even }}^{c ; \text { odd }}=\left.C_{I}^{\alpha}\right|_{\text {even }} ^{\text {odd }} F_{\text {sym }}^{c ; \text { odd }} F_{I}^{s ; \text { even }}
$$

where again $F_{\text {sym }}^{c ; \text { odd }}$ can be obtained from Eq. (23).

Notice that $\left.C_{I}^{\alpha}\right|_{\text {even }} ^{\text {odd }}$ corresponds to the perturbative case, see Eqs. (21), (23), (24) and Ref. [26]. Straightforward calculation gives

$$
\left.C_{I}^{u}\right|_{\text {odd }} ^{\text {even }}=-\frac{4}{9},\left.\quad C_{I}^{d}\right|_{\text {odd }} ^{\text {even }}=-\frac{8}{9}
$$

and

$$
\left.C_{I}^{u}\right|_{\text {even }} ^{\text {odd }}=-\frac{16}{9},\left.\quad C_{I}^{d}\right|_{\text {even }} ^{\text {odd }}=+\frac{4}{9} .
$$

Therefore, in the instanton approach the total expression for the Sivers function has two contributions of a very different origin, which sum up together:

$$
\begin{aligned}
f_{1 T}^{\perp \alpha}\left(x, k_{\perp}\right)_{I}= & M_{P} E_{P} \frac{f}{2 m^{*}} \tilde{N}^{4} \frac{1}{(2 \pi)^{5}} \int d^{2} \boldsymbol{q}_{\perp} \frac{1}{\boldsymbol{q}_{\perp}^{2}} G\left(q_{\perp} \rho_{c}\right) \\
& \times\left\{\left.C_{I}^{\alpha}\right|_{\text {odd }} ^{\text {even }}\left[t_{0}(k) t_{0}\left(k^{\prime}\right)+\frac{k_{z}}{k} t_{1}(k) t_{0}\left(k^{\prime}\right)+\frac{k_{z}}{k^{\prime}} t_{0}(k) t_{1}\left(k^{\prime}\right)+\frac{k^{2}-k_{\perp} q_{\perp} \cos \phi}{k k^{\prime}} t_{1}(k) t_{1}\left(k^{\prime}\right)\right]\right. \\
& \times \frac{q_{\perp} \cos \phi}{k_{\perp}} \int \kappa^{2} d \kappa d(\cos \theta)\left[t_{0}(\kappa) t_{0}\left(\kappa^{\prime}\right)+\frac{\kappa-q_{\perp} \cos \theta}{\kappa^{\prime}} t_{1}(\kappa) t_{1}\left(\kappa^{\prime}\right)\right]
\end{aligned}
$$




$$
\begin{aligned}
& +\left.C_{I}^{\alpha}\right|_{\text {even }} ^{\text {odd }}\left[\frac{k_{\perp}}{k} t_{1}(k) t_{0}\left(k^{\prime}\right)-\frac{k_{\perp}-q_{\perp} \cos \phi}{k^{\prime}} t_{0}(k) t_{1}\left(k^{\prime}\right)+\frac{k_{z} q_{\perp} \cos \phi}{k k^{\prime}} t_{1}(k) t_{1}\left(k^{\prime}\right)\right] \\
& \left.\times \frac{q_{\perp}}{k_{\perp}} \int \kappa^{2} d \kappa d(\cos \theta)\left[\cos \theta t_{0}\left(\kappa^{\prime}\right) t_{1}(\kappa)-\frac{\kappa \cos \theta-q_{\perp}}{\kappa^{\prime}} t_{0}(\kappa) t_{1}\left(\kappa^{\prime}\right)\right]\right\},
\end{aligned}
$$

where $\phi$ is the azimuthal angle of $\boldsymbol{q}_{\perp}$, and

$$
k_{z}=\frac{\omega(4 x-1)}{R_{0}}, \quad k=\sqrt{k_{z}^{2}+k_{\perp}^{2}}, \quad k^{\prime}=\sqrt{k_{z}^{2}+\left(\boldsymbol{k}_{\perp}-\boldsymbol{q}_{\perp}\right)^{2}} .
$$

Notice that to go from Eqs. (23), (30), (31), (27) to Eq. (37) we have used the identity

$$
\int d^{2} \boldsymbol{q}_{\perp} q_{\perp i} F\left(\boldsymbol{k}^{2}, \boldsymbol{q}_{\perp}^{2}, \boldsymbol{k}_{\perp} \cdot \boldsymbol{q}_{\perp}\right) \equiv \frac{k_{i}}{k_{\perp}^{2}} \int d^{2} \boldsymbol{q}_{\perp} \boldsymbol{k}_{\perp} \cdot \boldsymbol{q}_{\perp} F\left(\boldsymbol{k}^{2}, \boldsymbol{q}_{\perp}^{2}, \boldsymbol{k}_{\perp} \cdot \boldsymbol{q}_{\perp}\right),
$$

where $F$ is any function with the functional dependence shown and $i=x, y$. In this way, we can effectively take in Eqs. (23), (30), (31), $q_{\perp i} \rightarrow k_{i}\left(\boldsymbol{k}_{\perp} \cdot \boldsymbol{q}_{\perp}\right) / k_{\perp}^{2}$, and the numerator of the Sivers function, Eq. (27), becomes proportional to $k_{y}$, which simplifies, as it must be, with the overall factor $1 / k_{y}$ originally present in our expression.

\section{Numerical results}

In order to estimate the Sivers function for $u$ and $d$ valence quarks within the instanton approach proposed in this Letter, we do not make any attempt to fix the parameters of the instanton model by fitting available results on single spin asymmetries. Rather, we consider widely accepted values for these parameters, coming from a comprehensive application of the instanton model to nonperturbative QCD (see Ref. [17] for more detail): $\rho_{c} \approx 0.3 \mathrm{fm}, m^{*} \approx 170 \mathrm{MeV}$ and $n_{c} \approx 0.5 \mathrm{fm}^{-4}$. For the perturbative contribution we will take the following value of the strong coupling constant, $\alpha_{s} \approx 0.3$. This value is usually adopted for estimation of the one-gluon exchange contribution to SSA for HERMES kinematics (see e.g. Ref. [28]). This has to been taken into account when comparing our results with those of Yuan [26].

Using these parameters, in Fig. 2 we present the instanton and the perturbative, one-gluon exchange, contributions to the first moment of the Sivers function,

$$
f_{1 T}^{\perp(1) \alpha}(x)=\int d^{2} \boldsymbol{k}_{\perp}\left(\frac{k_{\perp}^{2}}{2 M_{P}^{2}}\right) f_{1 T}^{\perp \alpha}\left(x, k_{\perp}\right),
$$

for $u$ and $d$ quarks, as a function of $x$. In Fig. 3, the Sivers function is shown as a function of $k_{\perp}$, at $x=0.3$. The main features of these results are that the instanton contribution is sizable and, in contrast with the purely perturbative case, it has the same (negative) sign and approximately the same magnitude for $u$ and $d$ quarks.

As a consequence, when summing the two independent perturbative and instanton terms to get the total contribution to the Sivers function, for the $d$-quark the two terms almost cancel each other, leading to a small, negative Sivers function. On the contrary, in

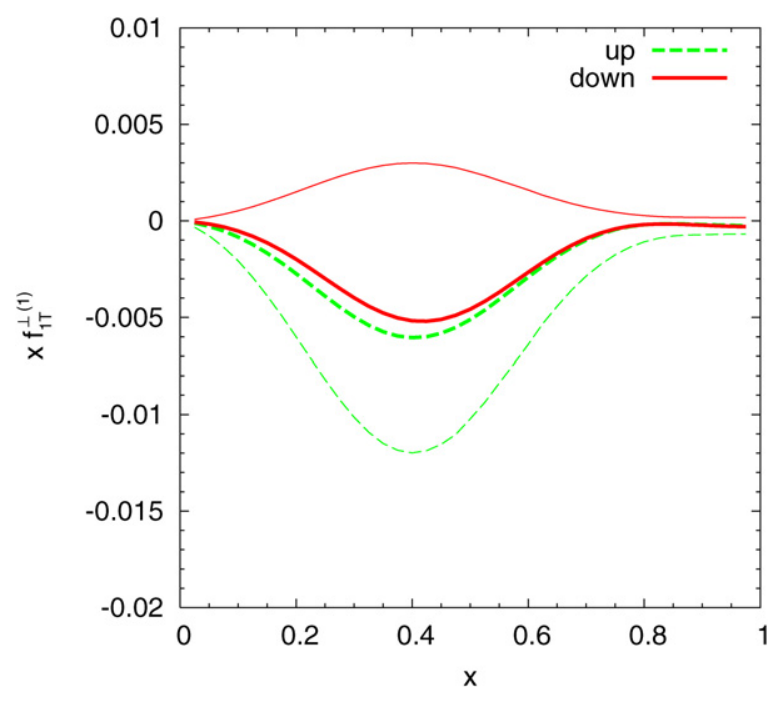

Fig. 2. The instanton (thick lines) and the one-gluon (thin lines) contributions to the first moment of the $d$-quark (solid) and $u$-quark (dashed) Sivers function, vs. $x$.

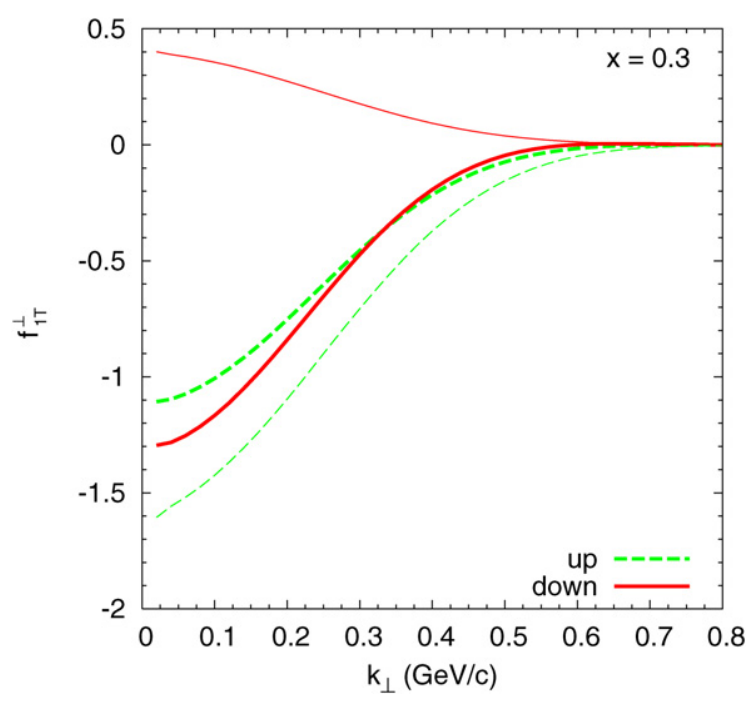

Fig. 3. The instanton and one-gluon contributions to the Sivers function at $x=0.3$ vs. $k_{\perp}(\mathrm{GeV} / c)$. Notations are the same as in Fig. 2. 


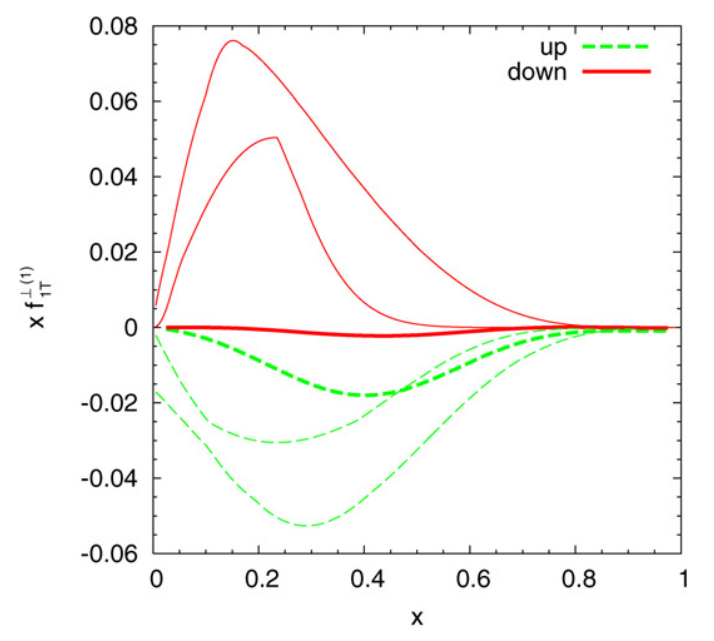

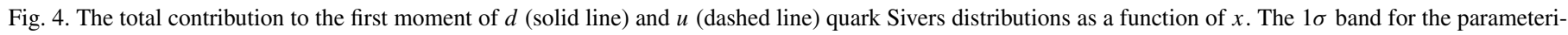
zations extracted by fitting the HERMES data (see first of Ref. [16]) is also shown.

the case of the $u$-quark Sivers function, the instanton contribution sums up with the perturbative one. This can be seen in Fig. 4 , where we also compare the total contribution to the first moment of the Sivers function with recent parameterizations obtained by fitting the results of the HERMES Collaboration for the single spin asymmetry in semi-inclusive DIS pion production [2], see first of Ref. [16]. From these results, one can observe that the present estimate of the instanton contribution to the valence-quark Sivers function compares reasonably well, for $u$ quarks, with parameterizations leading to a good description of the HERMES data in the valence-quark region, $x \geqslant 0.3$, where one can believe in the MIT-bag based calculations. On the other hand, for $d$ quarks our results are smaller than one could expect from data fitting and differ from those of other model calculations and available parameterizations.

\section{Conclusions}

In this Letter, the instanton contribution to the valence-quark Sivers function has been estimated, adopting the MIT bag model for the nucleon wave function. Our results show that this contribution can be sizable, since it is not suppressed by powers of the strong coupling constant. The specific flavour dependence of the instanton contribution, as compared to that of the perturbative one, leads to a large, negative total contribution to the $u$-quark Sivers function. On the other hand, it suppresses the total contribution in the $d$-quark case. However, several points should be kept in mind here:

(a) We have used widely accepted values for the basic parameters of the instanton model. These parameters are known with relatively large uncertainties which reflect into the results of our calculations. As an example, using the Diakonov and Petrov instanton liquid model estimate for the quark anomalous chromomagnetic moment (see e.g. Ref. [18]) would lead to an almost twice larger instanton contribution.

(b) The value adopted for the strong coupling constant plays a role in the interplay between the purely perturbative and the instanton terms, since the first contribution is proportional to $\alpha_{s}$ while the second one depends on it in a more involved and indirect way. Changing the effective value of $\alpha_{s}$, which is not well-known a priori, would therefore modify in particular the $d$-quark case.

(c) Due to the effective instanton size, the instanton contribution could in principle lead to a different $k_{\perp}$ dependence of the Sivers function as compared to the purely perturbative case. In our results, however, the two contributions show a very similar behaviour in this respect. This is because in this first simplified approach the effective $k_{\perp}$ dependence of the Sivers function is basically determined by the MIT bag model wave functions rather than by specific instanton properties.

(d) There could be in principle additional instanton contributions to the Sivers function, coming from more complex Feynman diagrams, involving e.g. the spectator diquark as a whole. Moreover, these contributions might be flavour-dependent, leading to different changes in the $u$ - and $d$-quark Sivers function.

A detailed treatment of all these points is beyond the scope of this Letter, which was mainly intended to show that instanton effects may be relevant for the Sivers distribution. A deepest study of instanton contributions to the Sivers function, including additional diagrams and based on more elaborated models for the nucleon wave function is in progress [32].

\section{Acknowledgements}

We are very grateful to Feng Yuan for enlightening discussions. We also thank D.I. Diakonov, A.E. Dorokhov, K. Goeke, W.-D. Nowak, P.V. Pobylitsa, M.V. Polyakov, A.V. Prokudin, A.E. Radzhabov, and N. Stefanis for fruitful critical comments. I.O.C. is 
grateful to Ulf-G. Meissner and B. Metsch for hospitality at Bonn University in the final stage of this work. N.I.K. is grateful to the School of Physics, SNU, and especially to Prof. Dong-Pil Min for their warm hospitality. This work is partially supported by: The Heisenberg-Landau program and the Grant of Russian Foundation for Basic Research, No. RFBR-04-02-16445 (I.O.C. and N.I.K.); the Russian President's Grant No. 1450-2003-2, and the Alexander von Humboldt-Stiftung and DFG (I.O.C.); the Brain Pool program of Korea Research Foundation through KOFST, Grant No. 042T-1-1 (N.I.K.); the European Community-Research Infrastructure Activity under the FP6 "Structuring the European Research Area" programme, HadronPhysics, Contract No. RII3CT-2004-506078 (U.D. and F.M.).

\section{References}

[1] A. Airapetian, et al., HERMES Collaboration, Phys. Rev. Lett. 84 (2000) 4047; A. Airapetian, et al., HERMES Collaboration, Phys. Rev. D 64 (2001) 097101.

[2] A. Airapetian, et al., HERMES Collaboration, Phys. Lett. B 622 (2005) 14.

[3] V.Y. Alexakhin, et al., COMPASS Collaboration, Phys. Rev. Lett. 94 (2005) 202002.

[4] S.S. Adler, et al., PHENIX Collaboration, Phys. Rev. Lett. 95 (2005) 202001.

[5] J. Adams, et al., STAR Collaboration, Phys. Rev. Lett. 92 (2004) 171801.

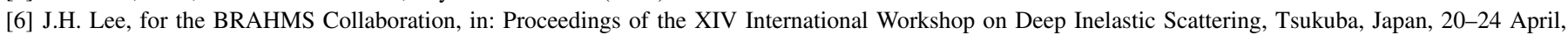
2006.

[7] See, e.g., S.B. Nurushev, Int. J. Mod. Phys. A 12 (1997) 3433;

K. Heller, in: Proceedings of the 12th International Symposium on High-Energy Spin Physics, World Scientific, Singapore, 1996 , p. 23.

[8] D.W. Sivers, Phys. Rev. D 41 (1990) 83;

D.W. Sivers, Phys. Rev. D 43 (1991) 261.

[9] J.C. Collins, Nucl. Phys. B 396 (1993) 161.

[10] J.w. Qiu, G. Sterman, Phys. Rev. Lett. 67 (1991) 2264.

[11] S.J. Brodsky, D.S. Hwang, I. Schmidt, Phys. Lett. B 530 (2002) 99.

[12] J.C. Collins, Phys. Lett. B 536 (2002) 43.

[13] A.V. Efremov, Ann. Phys. 13 (2004) 651.

[14] P.J. Mulders, R.D. Tangerman, Nucl. Phys. B 461 (1996) 197;

P.J. Mulders, R.D. Tangerman, Nucl. Phys. B 484 (1997) 538, Erratum;

D. Boer, P.J. Mulders, Phys. Rev. D 57 (1998) 5780.

[15] M. Anselmino, M. Boglione, F. Murgia, Phys. Lett. B 362 (1995) 164; M. Anselmino, M. Boglione, F. Murgia, Phys. Rev. D 60 (1999) 054027; M. Anselmino, M. Boglione, U. D’Alesio, E. Leader, S. Melis, F. Murgia, Phys. Rev. D 73 (2006) 014020.

[16] M. Anselmino, M. Boglione, U. D’Alesio, A. Kotzinian, F. Murgia, A. Prokudin, Phys. Rev. D 72 (2005) 094007; J.C. Collins, A.V. Efremov, K. Goeke, S. Menzel, A. Metz, P. Schweitzer, Phys. Rev. D 73 (2006) 014021; W. Vogelsang, F. Yuan, Phys. Rev. D 72 (2005) 054028; M. Anselmino, et al., hep-ph/0511017.

[17] T. Schäfer, E.V. Shuryak, Rev. Mod. Phys. 70 (1998) 323.

[18] D. Diakonov, Prog. Part. Nucl. Phys. 51 (2003) 173.

[19] A. Ringwald, F. Schrempp, Phys. Lett. B 459 (1999) 249.

[20] A.E. Dorokhov, N.I. Kochelev, Y.A. Zubov, Int. J. Mod. Phys. A 8 (1993) 603.

[21] A.E. Dorokhov, I.O. Cherednikov, Ann. Phys. 314 (2004) 321;

A.E. Dorokhov, I.O. Cherednikov, Phys. Rev. D 67 (2003) 114017;

A.E. Dorokhov, I.O. Cherednikov, Phys. Rev. D 66 (2002) 074009.

[22] N.I. Kochelev, Phys. Lett. B 426 (1998) 149.

[23] N.I. Kochelev, JETP Lett. 72 (2000) 481.

[24] D. Ostrovsky, E.V. Shuryak, Phys. Rev. D 71 (2005) 014037.

[25] P. Hoyer, M. Jarvinen, JHEP 0510 (2005) 080.

[26] F. Yuan, Phys. Lett. B 575 (2003) 45.

[27] L.P. Gamberg, G.R. Goldstein, K.A. Oganessyan, Phys. Rev. D 67 (2003) 071504.

[28] A. Bacchetta, A. Schaefer, J.J. Yang, Phys. Lett. B 578 (2004) 109.

[29] A. Bacchetta, U. D’Alesio, M. Diehl, C.A. Miller, Phys. Rev. D 70 (2004) 117504.

[30] A.V. Belitsky, X. Ji, F. Yuan, Nucl. Phys. B 656 (2003) 165; X. Ji, F. Yuan, Phys. Lett. B 543 (2002) 66.

[31] P. Faccioli, E.V. Shuryak, Phys. Rev. D 64 (2001) 114020.

[32] I.O. Cherednikov, U. D’Alesio, N.I. Kochelev, F. Murgia, in preparation. 